\title{
Gestational Dermatosis Shortly after Implantation Associated with Parental Class II HLA Compatibility and Maternal Immune Activation: Preliminary Report of a Prospective Case Series
}

\author{
Norbert Gleicher $^{a, c} \quad$ David H. Barad ${ }^{a}$ b \\ ${ }^{a}$ Center for Human Reproduction - New York and Foundation for Reproductive Medicine, New York, N.Y., \\ ${ }^{b}$ Departments of Epidemiology and Social Medicine as well as Obstetrics, Gynecology and Women's Health, \\ Albert Einstein College of Medicine, Bronx, N.Y., and 'Department of Obstetrics, Gynecology and Reproductive \\ Sciences, Yale University School of Medicine, New Haven, Conn., USA
}

\section{Key Words \\ Dermatosis of pregnancy $\cdot$ Skin rash . Implantation rash $\cdot$ Histocompatibility antigens · Autoimmunity $\cdot$ Immune activation · Pregnancy $\cdot$ Infertility}

\begin{abstract}
Background: Pregnancy represents a semiallograft, subject to similar immune responses as allogeneic organ transplants. Tolerance of pregnancy appears best with maximal class II HLA heterogeneity between mother and father, while compatibilities are associated with increased pregnancy loss and maternal autoimmunity. Tolerance abnormalities often involve skin reactions. Abnormalities in tolerance of the fetal graft may do the same. Objective: To define the characteristics of a newly described dermatosis in very early pregnancy. Methods: Prospective case series of 7 couples/12 clinical episodes. Results: The dermatosis was observed in 7 out of 285 women undergoing in vitro fertilization (IVF; $2.5 \%$; $95 \% \mathrm{Cl} 0.66-$ $4.26 \%$ ) and in 12 out of 277 total IVF cycles reaching embryo transfer $(4.3 \% ; 95 \% \mathrm{Cl}$ 1.93-6.73\%). Prior to IVF all women reported autoimmune clinically significant allergies. All but 1 couple demonstrated class II HLA compatibility. Two of 4 pregnancies miscar-
\end{abstract}

ried. All rashes erupted within days from embryo implantation. Conclusions: The 'implantation rash' reported here is uncommon but not rare. It may be the consequence of abnormal maternal immune responses to embryo implantation in women with prior immune activation, associated with class II HLA compatibility between parents. Further prospective studies are required to better define this condition.

Copyright $\odot 2011$ S. Karger AG, Basel

\section{Introduction}

Dermatoses of pregnancy are skin conditions restricted to the gestational period $[1,2]$. Only one dermatosis, the so-called prurigo of pregnancy, is exclusively limited to the first trimester of pregnancy, and no dermatoses have ever been described as being restricted to the immediate postimplantation period (table 1) [2].

We here describe such a dermatosis, previously over 15 years incidentally observed on rare occasions by one of the authors (N.G.), but never before systematically investigated. The accidental postfactum discovery that an affected patient and her husband shared class II HLA antigens to a significant degree (tested elsewhere prior to presentation to our center) led to the here reported prospective case accumulation over 22 months between October 2008 and July 2010.

This experience allows for a preliminary analysis of patient characteristics and treatment responses, and permits limited inferences about pathophysiology of this previously unreported dermatosis of pregnancy.

\section{Methods}

\section{Prevalence of Observation}

The 7 patients presented here represent $2.5 \%$ (95\% CI $0.66-4.26 \%$ ) of 285 women who came for in vitro fertilization (IVF) to our center between October 2008 and July 2010. They, in total, experienced 12 rash episodes during 14 IVF cycles (85.7\%), of which 12 were observed at our center in 277 cycles reaching embryo transfer $(4.3 \%$; 95\% CI 1.93-6.73\%) and 2 occurred during prior IVF cycles elsewhere.

Both authors contributed equally to the study and completion of the manuscript.

\section{KARGER}

Fax +4161306 1234 E-Mail karger@karger.ch www.karger.com
(C) 2011 S. Karger AG, Basel

$1018-8665 / 11 / 2223-0206 \$ 38.00 / 0$

Accessible online at: www.karger.com/drm
Norbert Gleicher

Center for Human Reproduction

21 East 69th Street

New York, NY 10021 (USA)

Tel. +1 212994 4400, E-Mail ngleicher@thechr.com 
This report describes an absolutely uniform skin rash in all reported patients, seen in infertility patients undergoing IVF a few days after embryo transfer, and either presumed or documented implantation. On rare occasions, anecdotally, one of the authors (N.G.) has been observing these rashes for approximately 15 years and found them responsive to corticosteroid therapy. A formal investigation was, however, never pursued. Though rare, these rashes were frequent enough to be given by staff at the center the acronym 'implantation rash'. Because often appearing shortly before a positive pregnancy test following IVF, appearance of the rash was considered by staff a positive sign in predicting pregnancy.

In this series a first rash was observed in October of 2008 in a woman who, post factum, notified us that she and her husband had previously been informed at another fertility center that they shared class II HLA antigens. Record review revealed that they, indeed, shared 2 class II antigens (table 3, patient 3). This observation led to the prospective accumulation of this case series of 'implantation rashes' over the ensuing 22 months.

\section{Description of Rash}

Rashes were self-reported by patients and photographed by either patients or medical staff. It is therefore possible, and even likely, that at least some milder cases may have gone unreported and, consequently, unrecognized. Rashes were uniformly nonpruritic reddish and papular, with small vesicular appearance, always only involving the neck, upper trunk, from the back to front of the chest and, often, the upper arms (fig. 1; photographs of rashes from other patients are available upon request from the authors).

\section{Possible Differential Diagnoses}

This prospective case accumulation suffers from a number of potential weaknesses: (i) not all reported episodes were observed and/or documented by a physician, as some were self-reported by patients; (ii) the skin eruptions were not subject to formal reviews by dermatologists, though 2 of the patients obtained dermatology consultations, received no specific diagnosis and were only told that 'the rash would go away'; (iii) the skin eruptions were therefore not subject to skin biopsies either.

Gestational Dermatosis Shortly after Implantation

Table 1. Dermatoses of pregnancy

Dermatosis

Time of occurrence

in pregnancy

Pruritic urticarial papules and plaques of pregnancy

Prurigo of pregnancy

Intrahepatic cholestasis of pregnancy

Pemphigoid gestationis

Impetigo herpetiformis

Pruritic folliculitis of pregnancy

3rd trimester

all trimesters ${ }^{\mathrm{a}}$

3rd trimester ${ }^{b}$

2nd, 3rd trimesters ${ }^{c}$

2nd, 3rd trimesters

2nd, 3rd trimesters

This table is mostly based on Tunzi and Gray [1].

${ }^{a}$ Can be prolonged, extending weeks to months into the postpartum period. ${ }^{b} \mathrm{Has}$ been associated with HLA-A31 and HLA-B8 in the mother. ${ }^{c}$ Established autoimmune disease, linked to HLA-DR3 and HLA-DR4.

Accordingly, potential differential diagnoses have to be considered carefully. Though all observed rashes were nonpruritic, the possibility of iatrogenic/atopic skin reactions had to be considered. Therefore, chart reviews placed special emphasis on whether the timing of the first occurrence was linked to the onset of treatments with new medications. However, no such associations were noted since the closest medication changes had taken place at least 8 days before the onset of symptoms.

Since the observed skin eruptions resembled features of acne vulgaris, special attention was paid to the treatment starts of 2 routine medications in the center's IVF protocol, known to be associated with a risk for acne vulgaris. (i) Patients with diminished ovarian reserve receive supplementation with dehydroepiandrosterone (DHEA) [3], but this treatment is initiated at least 6 weeks before the start of any IVF cycle, 9-10 weeks before first dermatological symptoms were noted. All patients in this case series suffered from severe diminished ovarian reserve and were therefore supplemented with DHEA. (ii) All of the center's patients also receive 10 mg of prednisone daily during IVF, starting with cycle start. This means that by the time rashes were reported, patients had already been, under prednisone treatment for at least 3 weeks on average. In addition, it must be noted that increases in prednisone dose improved symptoms in all patients, while reduction in prednisone dose - in at least 1 patient - indeed aggravated the severity of the rash. Like DHEA, prednisone is therefore only very unlikely to cause the dermatoses reported here, and no other medication used in the center's IVF protocols is known to be associated with skin reactions.

\section{HLA Typing}

The women so diagnosed were investigated for common historical denominators in medical history, laboratory findings and demographic data. In addition, both partners underwent HLA typing for class I (HLA-A, -B, -C) and class II (HLADR, -DQ) by PCR and hybridization with sequence-specific oligonucleotide probes carried out by a specialty laboratory (Quest Diagnostics, Nichols Institute Chantilly, Chantilly, Va., USA).

\section{Institutional Review Board}

At the time of initial consultation, patients at our center sign a universal informed consent, which allows for extraction of data from their medical records for research purposes as long as confidentiality of data is maintained and the identity of patients remains protected. In addition, both partners signed a specific 'experimental' consent for HLA typing. These blood tests were offered free of charge.

\section{Results}

\section{Patient Characteristics}

Table 2 summarizes the 7 couples. The mean age at the time of presentation to our center was 39 years (range 30-44), and practically all ethnicities/races were represented amongst the afflicted patients (see 
Table 2. Patient summary

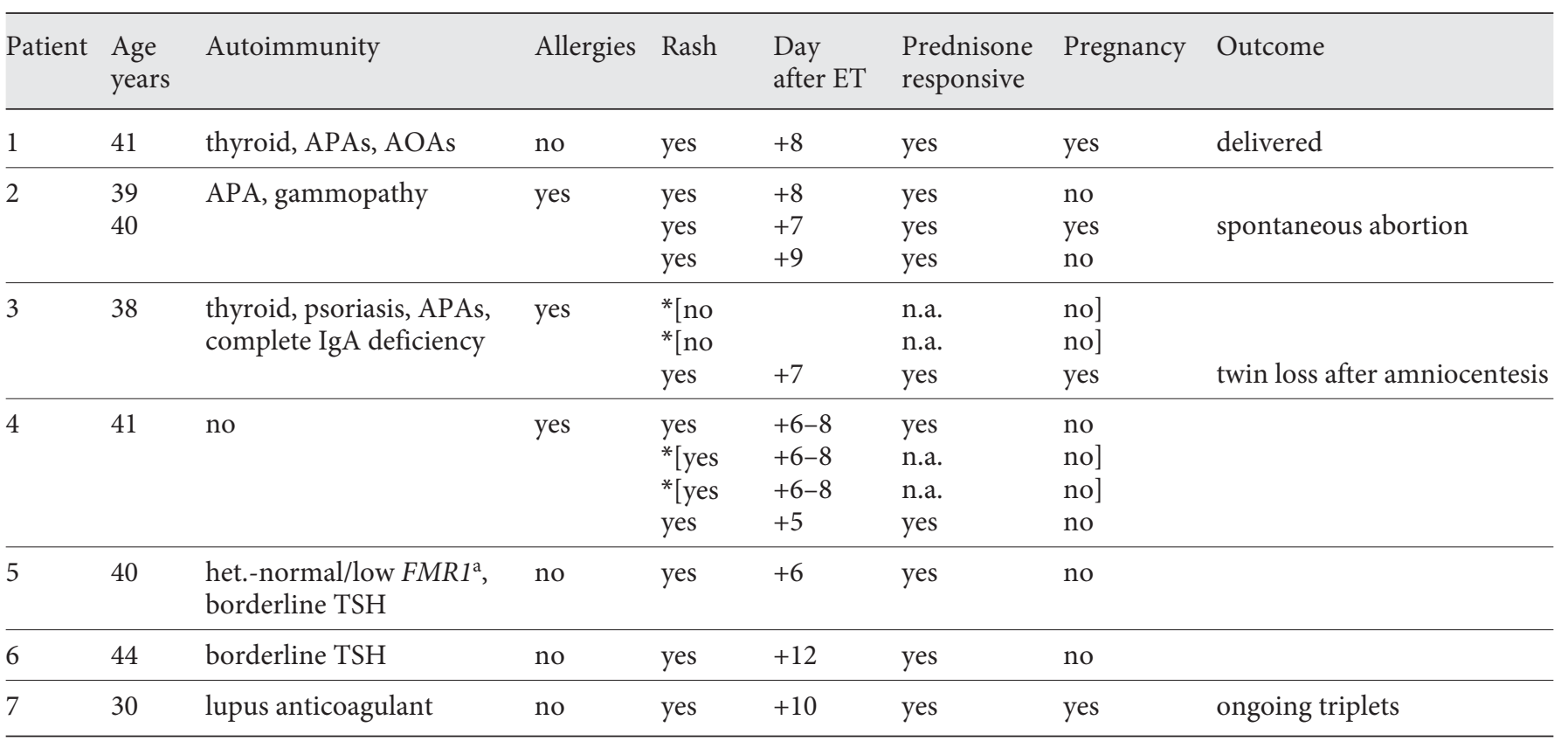

This dermatosis of pregnancy is seen in all races/ethnicities. Here represented are 2 Caucasian women, 1 Jewish Ashkenazi, 1 Asian Indian, 1 Chinese, 1 African and 1 Dominican patient.

$\mathrm{ET}=$ Embryo transfer; thyroid = thyroid autoimmune disease; APAs = antiphospholipid antibodies; AOAs = antiovarian antibodies; gammopathy = abnormal immunoglobulin level (IgG, $\operatorname{IgM}, \operatorname{Ig} \mathrm{A}) ;$ n.a. = not assessed; het. = heterozygous; $\mathrm{TSH}=$ thyroid-stimulating hormone; * [ ] denotes IVF cycle performed prior to presentation to our center.

Specific patient characteristics: patient 1: autoimmunity flared with positive pregnancy test evidenced by higher APA titers and additional positive APAs; patient 3: diagnosed with subchorionic bleed, which is statistically associated with autoimmunity (for further details, see discussion section); pregnancy was lost at 17 weeks after amniocentesis due to premature rupture of membranes; it was a genetically normal twin pregnancy; patient 4: reported recent onset of sudden and severe environmental allergies; patient 6: developed rash when prednisone was tapered after negative pregnancy test.

a This FMR1 subgenotype is found in infertile women closely associated with the presence of autoimmunity [4]. footnote of table 2). Four out of 7 women demonstrated laboratory evidence of autoimmunity. Two amongst 3 without clear laboratory evidence demonstrated highnormal thyroid-stimulating hormone levels, and the third was 1 out of 3 patients with significant allergies.

\section{Response to Oral Corticosteroids}

The rash in all 7 patients improved with increasing prednisone dosage and, indeed, in 1 patient increased in severity after prednisone had been tapered.

Recurrence with Repeat Pregnancy

Three patients experienced more than 1 IVF cycle. In 2 of these patients, the rash reoccurred in all cycles at similar times. The third patient reported a rash only in her third IVF cycle.
Timing of Rash

Rashes occurred between days 5 and 12 after embryo transfer, approximately reflective of days 3-10 after the presumed implantation [5].

\section{Pregnancies}

None of our patients experienced a chemical pregnancy. Only 4 out of 14 IVF cycles led to clinical pregnancy (28.6\%), a disappointing finding considering the previously noted anecdotal impression that the 'implantation rash' was suggestive of high pregnancy chances. Considering that all but 1 female were at or above the age of 38 years, all demonstrated folliclestimulating hormone levels above 10.0 $\mathrm{mIU} / \mathrm{ml}$ (up to $37.2 \mathrm{mIU} / \mathrm{ml}$ ) and antimüllerian hormone levels between undetectable $(<0.1 \mathrm{ng} / \mathrm{ml})$ and $0.4 \mathrm{ng} / \mathrm{ml}$; this IVF pregnancy rate is, however, quite respectable.

More disappointingly, only 2 pregnancies are either already delivered or at advanced stages of pregnancy at the time of this report, for a likely live birth rate of only $14.3 \%$. A twin pregnancy was lost for iatrogenic reasons after amniocentesis, and a fourth pregnancy was lost by early spontaneous abortion.

\section{HLA Typing}

Table 3 summarizes class II HLA typing in all couples. As can be seen, all but 1 couple demonstrated a significant overlap in class II loci, a statistically highly unlikely finding. However, it needs to be pointed out that that study does not offer a control group amongst IVF patients who did not develop an 'implantation rash'. 
Table 3. Class II HLA typing of couples

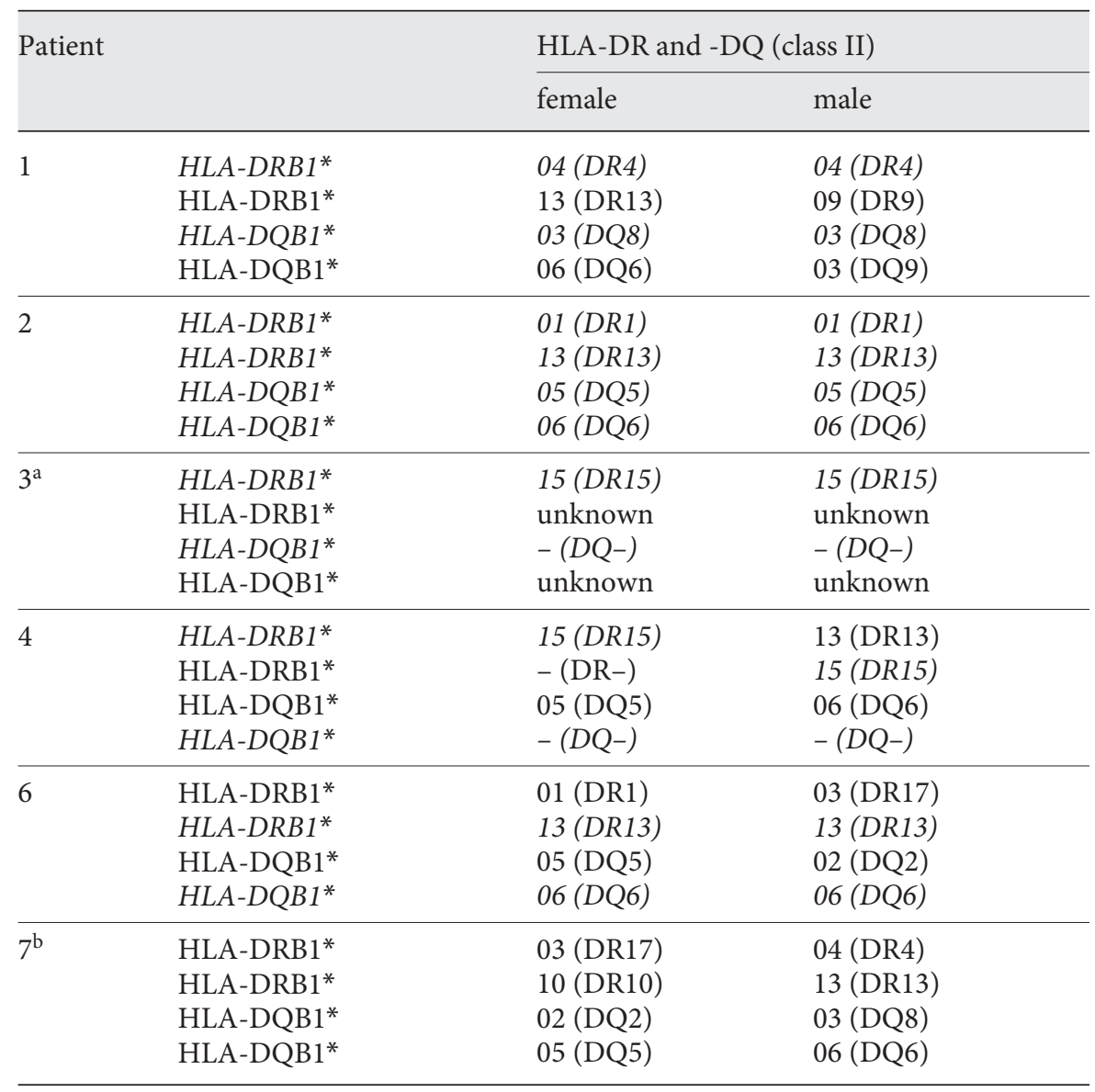

Serology equivalents are given in parentheses; - = homozygosity or unidentified. Italics denote matching loci between partners.

${ }^{a}$ Patient 3, the initial patient in the series, is the only one who was tissue typed at another fertility center prior to presentation to our center.

${ }^{\mathrm{b}}$ Only patient with no common locus with partner.

\section{Discussion}

The dermatosis described here is uncommon but not rare, with an approximate prevalence amongst infertility patients undergoing IVF of $2.5 \%(95 \% \mathrm{CI}$ $0.66-4.26 \%$ ) and a $4.3 \%$ incidence in IVF cycles reaching embryo transfer $(95 \% \mathrm{CI}$ $1.93-6.73 \%)$. It is characterized by early onset, 5-12 days after embryo transfer (310 days after implantation) [6], and follows a standard and uniform pattern of very selective areas of skin eruption on the neck, upper trunk and arms (fig. 1).

The exact nature of this rash can, ultimately, only be determined by skin biopsy. Therefore, a final determination of the pathophysiology of this dermatosis awaits further future investigations. Considering the relatively quick improvements of clinical presentation with increasing prednisone doses, we felt that in the reported cases a skin biopsy would not have been ethically appropriate without prior Institutional Review Board approval and appropriate study consents. The value of skin biopsies in assessing failure in tolerance after tissue transplants is actually quite limited [6].

In addition, as previously noted, differential diagnoses in this case series are limited. An allergic/atopic reaction appears unlikely since the rash was nonpruritic and does not appear associated with therapeutic introductions of new medications. It also seems unlikely that acne vulgaris would suddenly arise within a brief time period after embryo transfer and implantation [5], unrelated to DHEA supplementation or prednisone administration. The latter conclusion is also supported by the quick clinical improvements of the rash in all reported patients after prednisone doses had been increased. Acne vulgaris is, of course, not known to be responsive to corticosteroids.

Patient 1 suggested that appearance of the rash may be closely associated with flaring of autoimmune laboratory abnormalities. Since only antiphospholipid antibodies were monitored in the reported patients [7], absences of antiphospholipid antibodies not necessarily indicate that autoimmunity did not flare in the other 2 patients [8]. Indeed, a spontaneous pregnancy loss in patient 2 and a threatened twin pregnancy in patient 3 , characterized by vaginal bleeding and a subchorionic hematoma, could also point towards autoimmune activation. Subchorionic bleeds [9] and the risk of spontaneous pregnancy loss have been associated with autoimmunity $[7,8]$.

Parental class II histocompatibility, the most remarkable common clinical characteristic of the reported patients, is indeed fairly typically characterized by autoimmunity. Maternal autoimmunity is associated in pregnancy with 2 typical flare periods: a first in early pregnancy, associated with an increased miscarriage risk, and a second peri- and postpartum period, when autoimmune diseases tend to exacerbate $[7,8]$. Both represent time periods when the maternal immune system is exposed to previously unprecedented volumes of paternal HLA antigens, with implantation in early pregnancy, and peak fetal-maternal cell traffic at term and delivery [10-12].

The fetal-placental unit represents immunologically a semi-allograft, in normal pregnancy immunologically tolerated by the maternal immune system, though not always to the same degree. For example, success of tolerance depends on HLA compatibilities [11]. Inadequate tolerance will first surface clinically with implantation when, except for prior exposure to semen, the maternal immune system faces for the first time exposure to significant paternal HLA antigens. Allogeneic organ transplantation experience demonstrates that autoimmune responses and skin reactions are frequent manifestations of abnormal tolerance [11]. 

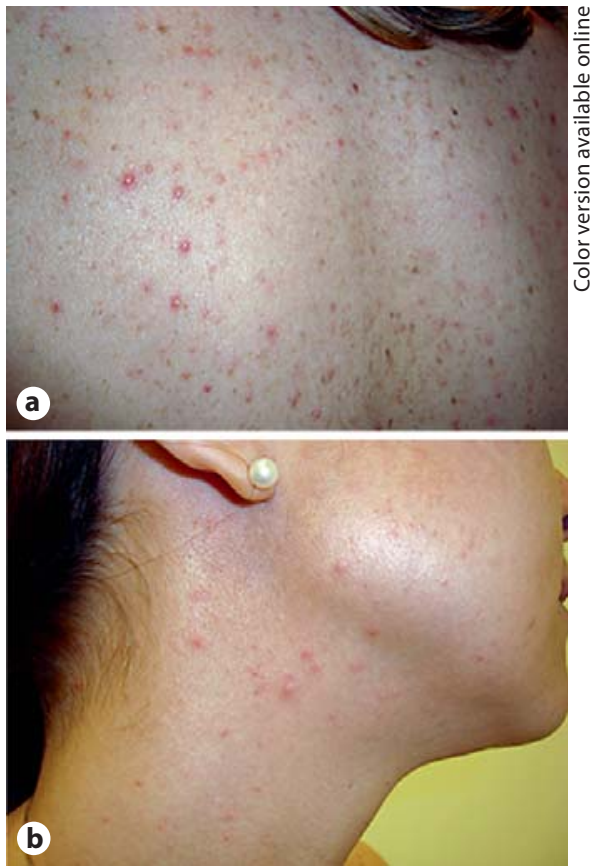

Fig. 1. Skin rash in patients 1 and 2. Rashes in all other patients looked identical, and photographic documentation of rashes in other patients is available from the authors upon request. a Rash on the back of patient 1 . b Rash on the neck and only the adjacent lower portion of the face in patient 2 .

In tissue transplantation such immune responses are often the consequence of either acute or chronic graft-versus-host disease. They require immunocompetent donor cells [13], which implanting embryos do, of course, not yet possess. Any immune response so shortly after implantation has therefore to represent an antiHLA response by the recipient host (i.e. the mother's immune system) against paternal HLA antigens.

Nelson et al. [14] demonstrated in association with rheumatoid arthritis that the maternal immune system will respond differently, depending on HLA compatibilities, with offspring representing paternal HLA. Close matches between maternal and paternal class II HLA favored autoimmune flares in pregnancy, characterized by exacerbations of rheumatoid arthritis. In contrast, dissimilarities in HLA favored abatement of disease activities during pregnancy.
Similarly, hyporesponsiveness to paternal HLA has been suggested to increase the miscarriage risk [15], and autoimmunity in early pregnancy has, of course, historically been closely associated with an increased miscarriage risk $[7,8]$. In combination, these observations suggest that induction of normal tolerance will succeed if paternal and maternal class II HLA loci vary to a sufficient degree, while close matches appear associated with autoimmune activation of the maternal immune system, as originally observed with rheumatoid arthritis [14].

All presented couples but 1 demonstrated excessive class II HLA compatibilities, suggesting that these women may be immunologically hyporesponsive to their partners and, therefore, may induce autoimmunity as part of inadequate maternal allograft tolerance towards the fetal-placental unit.

Women who develop the dermatosis of very early conception described here may, therefore, also have to be considered at risk of spontaneous pregnancy loss. Ober et al. [16] support such a conclusion by suggesting that HLA-DQA1-compatible fetuses may be aborted very early in pregnancy. Takakuwa et al., who were initially skeptical [17], more recently concluded [18] that compatibility of HLA class II antigens between husband and wife may be involved in the genesis of unexplained recurrent pregnancy loss.

Early appearance of dermatological symptoms will attract attention in infertile women under close observation but can easily be missed in women who conceive spontaneously and, at the very early stages of pregnancy, are often still unaware of conception. The fact that our cases occurred in infertile women may therefore represent a selection bias. It remains to be determined whether the known increased prevalence of autoimmunity in infertile women $[7,19,20]$ is responsible for the clustering of cases observed here.

In summary, this case series likely represents a previously unreported dermatosis of very early pregnancy. In principle, it appears associated with implantation, prior to the first positive pregnancy test, in women with a significant (auto)immune system activation and, likely, class II HLA compatibility between parents.

Clinical presentation and good therapeutic response to corticosteroid therapy suggest that this dermatosis may be the consequence of abnormal and/or inade- quate induction of fetal-placental tolerance by the maternal immune system. The resultant skin rash may therefore, akin to rashes observed in association with graft-versus-host disease and allogeneic organ transplant rejection, represent a response of the maternal immune system to paternal HLA antigens. Thus, occurrence of this dermatosis may also point towards an increased early miscarriage risk [15].

Our data are preliminary in nature and only observational. To confirm the reported findings of a new dermatosis of very early pregnancy and its proposed pathophysiology, further cases need to be prospectively accumulated, demonstrating an association with HLA class II overlaps between mother and father as well as maternal autoimmunity. Most importantly, however, skin biopsies will have to be performed to demonstrate similarities with reported skin lesions in allogeneic graftversus-host disease and graft rejection.

\section{Disclosure Statement}

Both authors are co-inventors and owners of a number of awarded and pending patent applications, including claims of beneficial effects on ovarian function and miscarriage rates from supplementation with DHEA administered to women with diminished ovarian reserve. N.G. is owner of the fertility center where the cases reported here received treatments. Both investigators have received speaker honoraria, research grant support and travel cost reimbursements from various pharmaceutical companies. However, none of these companies relate or have been associated in any way to the reported data.

\section{References}

-1 Tunzi M, Gray GR: Common skin conditions during pregnancy. Am Fam Physician 2007;75:211-218.

$>2$ Roth MM: Pregnancy dermatoses: diagnosis, management and controversies. Am J Clin Dermatol 2011;12:25-41.

-3 Barad D, Brill H, Gleicher N: Update on the use of dehydroepiandrosterone supplementation among women with diminished ovarian function. J Assist Reprod Genet 2007;24: 629-634. 
4 Gleicher N, Weghofer A, Lee IH, Barad DH: FMR1 genotype with autoimmunity-associated polycystic ovary-like phenotype and decreased pregnancy chance. PLoS One 2010;5: e15303.

5 Tur-Kaspa I, Confino E, Dudkiewicz AB, Myers SA, Friberg J, Gleicher N: Ovarian stimulation protocol for in vitro fertilization with gonadotropin-releasing hormone agonist widens the implantation window. Fertil Steril 1990;53:859-864.

6 Kohler S, Hendrickson MR, Chao NJ, Smoller BR: Value of skin biopsies in assessing prognosis and progression of acute graftversus-host disease. Am J Surg Pathol 1997; 21:988-996.

7 Gleicher N, Pratt D, Dudkiewicz A: What do we really know about autoantibody abnormalities and reproductive failure: a critical review. Autoimmunity 1993;16:115-140.

8 Gleicher N, Weghofer A, Barad D: Female infertility due to abnormal autoimmunity: frequently overlooked and greatly underappreciated. Part I. Expert Rev Clin Obstet Gynecol 2007;2:453-464.
-9 Baxi LV, Pearlstone MM: Subchorionic hematomas and the presence of autoantibodies. Am J Obstet Gynecol 1991;165:14231424.

10 Gleicher N: Why much of the pathophysiology of preeclampsia/eclampsia has to be 'autoimmune' in nature. Am J Obstet Gynecol 2007;196:5.31-5.37.

11 Gleicher N: Graft-versus-host disease and immunological rejection: implications for diagnosis and treatments of pregnancy complications. Expert Rev Obstet Gynecol 2008; 3:37-49.

12 Gleicher N, Elkayam U: Peripartum cardiomyopathy, an autoimmune manifestation of allograft rejection? Autoimmun Rev 2009;8: 384-387.

13 Feng G, Chan T, Wood KJ, Bushella: Donor reactive regulatoy $\mathrm{T}$ cells. Curr Opin Organ Transplant 2009;14:432-438.

14 Nelson JK, Hughes KA, Smith AG, Nisperos BB, Branchaud AM, Hansen JA: Maternalfetal disparity in HLA class II alloantigens and pregnancy-induced amelioration of rheumatoid arthritis. N Engl J Med 1993;329: 466-471.

15 Carp HJ, Toder V, Mashiach S: Immunotherapy of habitual abortion. Am J Reprod Immunol 1992;28:281-284
16 Ober C, Steck T, van der Ven K, Billstrand C, Messer L, Kwak J, Beaman K, Beer A: MHC class II class compatibility in aborted fetuses and term infants of couples with recurrent spontaneous abortion. J Reprod Immunol 1993;25:195-207.

17 Takakuwa K, Higashino M, Ueda H, Yamada K, Asano K, Yasuda M, Ishii S, Kazama Y, Tanaka K: Significant compatibility does not exist at the HLA-DQB gene locus in couples with unexplained recurrent abortions. Am J Reprod Immunol 1992;28:12-16.

18 Takakuwa K, Honda K, Yokoo T, Hatava I, Tamura M, Tanaka K: Molecular genetic studies on the compatibility of HLA class II alleles in patients with unexplained recurrent miscarriage in Japanese population. Clin Immunol 2006;118:101-107.

19 Gleicher N, el-Roeiy A: The reproductive autoimmune failure syndrome. Am J Obstet Gynecol 1988;159:223-227.

20 Geva E, Amit A, Lerner-Geva L, Lessing JB: Autoimmunity and reproduction. Fertil Steril 1997;67:599-611. 